\title{
Calculation of the thermokinetic EMF during a reverse phase transformation in TiNi alloys
}

\author{
V. V. Rubanik, A. V. Lesota ${ }^{\dagger}$, V. V. Rubanik Jr. \\ †ann20zv@tut.by \\ ${ }^{1}$ Institute of Technical Acoustics NAS of Belarus, 13 Lyudnikova ave., 210023, Vitebsk, Belarus \\ ${ }^{2}$ Vitebsk State Technological University, 72 Moskovsky ave., 210035, Vitebsk, Belarus
}

\begin{abstract}
It is known that a thermokinetic electromotive force (EMF) in TiNi is induced due to thermoelastic phase transformations in a local zone. The initiation of these transformations is possible during the movement of both the local heating and cooling zones along a TiNi alloy sample. Local heating of the conductor results in a reverse phase transfomation $\left(T \geq A_{s}\right)$, while cooling results in a direct phase transfomation $\left(T \leq M_{s}\right)$. Since the amount of the martensite phase in TiNi based materials is determined by the temperature of a sample, it has been assumed that the value of thermokinetic EMF depends on the temperature in the heating zone too. Therefore, the aim of the present work was to determine the dependence of the thermokinetic EMF on heating temperature. In this paper, we propose a physical model, in which the thermokinetic EMF is initiated as a result of the potential difference in areas with direct and reverse phase transformations. These areas occur during the motion of a heating zone $\left(T>A_{s}\right)$ along the TiNi alloy. Mathematical relationships which allow for a calculation of thermokinetic EMF initiated by reverse phase transformation in TiNi alloys and its dependence on temperature in the heating zone have been obtained. It has been found that thermokinetic EMF is induced when alloy temperature in the heating zone reaches $A_{s}$ and then grows to $0.23 \mathrm{mV}$ with increasing of alloy temperature up to $A_{f}$. A further increase of the heating zone temperature does not affect the value of thermokinetic EMF. The results of calculations of thermokinetic EMF in TiNi alloys during reverse phase transformation obtained are in good agreement with experimental data.
\end{abstract}

Keywords: TiNi alloys, thermokinetic EMF, reverse transformation.

\section{Расчет термокинетической ЭДС при обратном фазовом переходе в никелиде титана}

\author{
Рубаник В. В., Лесота А. В. ${ }^{\dagger}$ Рубаник В. В. мл. \\ †ann20zv@tut.by
}

\begin{abstract}
${ }^{1}$ Институт технической акустики НАН Беларуси, пр-т Людникова 13, 210023, Витебск, Беларусь
${ }^{2}$ Витебский государственный технологический университет, Московский пр-т 72, 210035, Витебск, Беларусь
\end{abstract}

Известно, что наведение термокинетической ЭДС в никелиде титана происходит при реализации термоупругих фазовых превращений в локальной зоне. Причем возникновение термокинетической ЭДС возможно как при перемещении участка нагрева вдоль протяженного TiNi образца, так и локального участка охлаждения. При этом нагрев в локальной зоне проводника обуславливает обратный фазовый переход ( $\left.T \geq A_{н}\right)$, а охлаждение - прямой $\left(T \leq M_{н}\right)$. Так как в материалах на основе никелида титана количество мартенситной фазы определяется температурой образца, было предположено, что и величина термокинетической ЭДС зависит от величины температуры в зоне нагрева. Определение зависимости величины наводимой термокинетической ЭДС от температуры при нагреве и являлось целью данной работы. В работе предложена физическая модель, согласно которой термокинетическая ЭДС инициируется в результате возникновения разности потенциалов на участках с прямым и обратным фазовым превращениями, возникающими при движении зоны нагрева вдоль TiNi сплава. Получены математические выражения позволяющие рассчитать величину термокинетеской ЭДС при обратном фазовом превращении в сплаве TiNi, а также зависимость данной величины от температуры в зоне нагрева. Показано, что термокинетическая ЭДС возникает при достижении температуры в зоне нагрева $A_{н}$ и увеличивается до 0,23 мВ с повышением температуры до $A_{k}$, дальнейшее повышение температуры на величину термокинетической ЭДС не влияет. Результаты расчетов величины термокинетической ЭДС в сплавах TiNi при обратном фазовом превращении хорошо согласуются с экспериментальными данными.

Ключевые слова: TiNi сплавы, термокинетическая ЭДС, обратное превращение. 


\section{1. Введение}

В настоящее время изучению термоэлектрических явлений уделяется большое внимание. Это связано с тем, что они находят широкое практическое применение в различных областях техники и могут проявляться как в разнородных, так и в однородных материалах. Примером возникновения электродвижущей силы (ЭДС) в однородных материалах служит наведение термокинетической ЭДС в результате перемещения зоны нагрева вдоль проволочного образца железа с постоянной скоростью [1]. Причиной возникновения такого рода термокинетической ЭДС является реализация фазового превращения в локальной зоне нагрева при температуре $700-800^{\circ} \mathrm{C}$. В ряде материалов фазовые превращения могут протекать при значительно более низких, вплоть до отрицательных, температурах, например, в сплавах с эффектом памяти формы. Наведение термокинетической ЭДС в никелиде титана происходит при реализации термоупругих фазовых превращениях, причем инициирование таких превращений возможно как при перемещении участка нагрева по проводнику $[2,3]$, так и локального участка охлаждения с постоянной скоростью [4]. При этом при нагреве в локальной зоне проводника происходит обратный фазовый переход (необходимо, чтобы в зоне нагрева температура достигала $A_{н}$ ), а при охлаждении - прямой (необходимо, чтобы температура на участке охлаждения снижалась до $M_{н}$ ).

Поскольку в материалах на основе никелида титана количество мартенситной фазы определяется температурой образца, было предположено, что и величина термокинетической ЭДС зависит от величины температуры в зоне нагрева. Определение зависимости величины наводимой термокинетической ЭДС от температуры при нагреве и являлось целью данной работы.

\section{2. Основная часть}

В процессе перемещения участка нагрева $\left(T>A_{\mu}\right)$ с постоянной скоростью вдоль протяженного TiNi образца (изначально находящегося в мартенситном фазовом состоянии) по обе стороны от участка нагрева будут протекать фазовые превращения. На переднем фронте в результате нагрева - обратное фазовое превращение, на заднем фронте в результате охлаждения нагретого участка, посредством теплообмена с окружающей средой, - прямое фазовое превращение. Предположено, что на участках с фазовыми превращениями возникает контактная разность потенциалов, обусловленная взаимодействием аустенитной и мартенситной фаз с различным значением плотности электронных состояний на уровне Ферми [5]. Таким образом, при движении зоны нагрева вдоль проволочного TiNi образца, на участках с прямым и обратным фазовыми превращениями, за счет возникновения контактной разницы потенциалов между аустенитом и мартенситом, будет возникать термо-ЭДС. Величина возникающей термо-ЭДС на участках с прямым и обратным фазовым превращением будет различна, в связи с разными значениями температур мартенситных переходов этих превраще- ний. Таким образом, величина термокинетической ЭДС, возникающая в результате движения участка нагрева $\left(T>A_{н}\right)$ вдоль TiNi образца, равна разнице значений термо-ЭДС возникающих на участках с прямым и обратным фазовым переходами.

Для расчета величины термокинетической ЭДС $(E)$ предложена следующая формула:

$$
\begin{aligned}
E\left(t, T_{\max }\right) & =\int_{x=0}^{x=x_{0}+v t}\left[\left(\frac{\partial S_{n p}(T(x, t))}{\partial x}\right) \cdot\left(T(x, t)-T^{0}\right) \cdot\left(1-\gamma_{o \delta p}\left(T_{\max }\right)\right)\right] d x- \\
& -\int_{x=x_{0}+v t}^{x}\left[\left(\frac{\partial S_{o \delta p}(T(x, t))}{\partial x}\right) \cdot\left(T(x, t)-T^{0}\right)\right] d x
\end{aligned}
$$

где $x_{0}$ - координата источника нагрева в начальный момент времени; $t$ - время; $T(x, t)$ - температура проволочного образца в точке с координатой $x$ в момент времени $t$; $v$ - скорость движения участка нагрева; $T^{0}-$ температура проволочного $\mathrm{TiNi}$ образца в невозмущенном состоянии; $S_{n p}, S_{\text {обр }}-$ коэффициент термо-ЭДС при прямом и обратном переходе; $\gamma_{\text {обр }}$ - доля мартенсита при обратном фазовом превращении.

В формуле 1 левый интеграл соответствует величине термо-ЭДС возникающей на участке с прямым фазовым превращением, в заданный момент времени, правый с обратным превращением. При нахождении термо-ЭДС (по формуле 5) возникающей на двухфазных участках посредством использования формул 3,4 учитывается доля TiNi образца претерпевающая прямой и обратный фазовый переход.

В расчетах необходимо также учитывать, что на участке с прямым переходом фазовое превращение будет претерпевать только та доля TiNi образца, которая совершила обратный фазовый переход при нагреве. Таким образом, доля TiNi образца, которая будет претерпевать прямой фазовый переход, зависит как от температуры, до которой охладится образец при конвекционном взаимодействии с окружающей средой (что учитывается при расчете формулы 5), так и от количества образованного аустенита при обратном фазовом превращении $\left(1-\gamma_{\text {oбp }}\left(T_{\max }\right)\right)$.

Так как термо-ЭДС возникает при контакте двух разнородных веществ и изменении температуры, то при движении зоны нагрева с температурой ниже температуры начала обратного фазового перехода $\left(T<A_{н}\right)$, термо-ЭДС в проволочном образце возникать не будет (образец находится в мартенситном состоянии). При движении зоны нагрева вдоль проволочного образца TiNi с температурой выше температуры конца обратного фазового перехода $\left(T>A_{\kappa}\right)$, участок зоны нагрева будет находиться в аустенитном состоянии. При этом, чем выше температура нагрева, тем больше градиент температуры около участка нагрева. При увеличении градиента температур будет увеличиваться ЭДС, возникающая в результате эффекта Бенедикса [6,7]. Так как величина данной ЭДС незначительна, ее значением можно пренебречь. В связи с тем, что при мартенситном превращении доля мартенситной фазы оказывается однозначной функцией температуры и не зависит от скорости нагрева в широком интервале значений этих скоростей [8], величина термокинетической ЭДС так же не должна зависеть от скорости движения зоны нагрева. 
Для расчета распределения температуры вдоль образца при движении зоны нагрева, рассмотрен случай, когда:

- в месте нахождения источника нагрева, нагрев проволочного образца до заданной температуры происходит мгновенно;

- движение источника нагрева вдоль проволочного образца происходит с постоянной скоростью;

- перенос тепловой энергии по длине образца, в обе стороны от зоны нагрева, осуществляется вследствие теплопроводности;

- имеет место конвекционный теплообмен образца с окружающей средой.

С учетом изложенных выше предположений распределение температуры по длине образца описывается неоднородным дифференциальным уравнением второго порядка в частных производных:

$$
\rho^{\prime} c \frac{\partial T}{\partial t}=\frac{\partial}{\partial x}\left(\sigma \frac{\partial T}{\partial x}\right)+Q\left(x+v_{0} t\right)-b\left(T^{e}-T^{w}\right)
$$

с начальными и краевыми условиями:

$$
\begin{array}{lc}
T(x, 0)=T^{w}, & 0 \leq x \leq x_{f} ; \\
T(0, t)=T\left(x_{f}, T\right)=T^{w}, & t>0 .
\end{array}
$$

Здесь $x_{f}$ - координата конца проволочного образца; $c, \rho^{\prime}$ - удельная теплоемкость и плотность материала образца соответственно; $\sigma-$ теплопроводность; $b=p a / S$, $p, S$ - периметр и площадь поперечного сечения проволоки, $a-$ коэффициент теплообмена с окружающей средой, $T^{e}$ - температура проволочного образца в заданный момент времени; $T^{w}$ - температура окружающей среды; $v_{0}$ - скорость движения участка нагрева; $Q-$ мощность внутреннего источника тепловыделения.

Локальный нагрев проводника приводит к обратному фазовому превращению, которое в свою очередь влияет на физические свойства TiNi сплава. T.e., входящее в уравнение (2) удельная теплоемкость будет являться функцией температуры. Для определения ее изменения использовали зависимость [9]:

$$
C(T)=C_{0}+C_{1}^{\prime}\left[\sin \left(2 \pi \frac{T-A_{s}}{A_{f}-A_{s}}-\frac{\pi}{2}\right)+1\right],
$$

где $C_{1}^{\prime}$ - константа материала.

При расчете распределения температур вдоль проволочного образца (рис. 1) задавали следующие параметры: скорость движения зоны нагрева 0,44 cм/с; характеристические температуры мартенситных переходов: $M_{н}=45^{\circ} \mathrm{C}, M_{\kappa}=33^{\circ} \mathrm{C}, A_{н}=59^{\circ} \mathrm{C}, A_{\kappa}=76^{\circ} \mathrm{C}$; длина участка источника нагрева $1 \mathrm{~cm}$.

Установлено, что распределение температуры вблизи зоны нагрева при ее движении устанавливается к 5 секунде, после чего распределение температуры остается постоянным во времени, изменяется только ее местоположение относительно проволочного образца (рис. 1а). На участках прямого и обратного фазовых превращений, видно, что изменение теплоемкости TiNi образца при фазовом превращении, вносит вклад в распределение температуры (рис. 1b).

Для описания зависимости доли мартенсита $\gamma$ от температуры $T$ при прямом $\gamma_{n p}$ и обратном $\gamma_{\text {обр }}$ мар- тенситных превращениях используем функции ЛиангаРоджерса [10]:

$$
\begin{array}{cc}
\gamma_{n p}(T)=\frac{1}{2}\left[\cos \left(a_{M}\left(T-M_{f}\right)\right)+1\right], & M_{f}<T<M_{s}, \\
\gamma_{o \delta p}(T)=\frac{1}{2}\left[\cos \left(a_{A}\left(T-A_{s}\right)\right)+1\right], & A_{s}<T<A_{f},
\end{array}
$$

где $a_{M}=\pi /\left(M_{s}-M_{f}\right), a_{A}=\pi /\left(A_{f}-A_{s}\right)$.

Для нахождения величины коэффициента термоЭДС в гетерофазных системах применима формула [11]:

$$
S(T)=\frac{\left(\rho(T) \sigma(T)-\rho_{2} \sigma_{2}\right)\left(S_{1}-S_{2}\right)+S_{2} \cdot\left(\rho_{1} \sigma_{1}-\rho_{2} \sigma_{2}\right)}{\rho_{1} \sigma_{1}-\rho_{2} \sigma_{2}} .
$$

В данной модели обобщенное удельное электрическое сопротивление $\rho$ и теплопроводность $\sigma$ рассматриваются как нормализованная сумма фазовых вкладов [12]:

$$
\rho(T)=\sum \frac{\gamma_{i}(T) \rho_{i} g_{i}(\rho)}{\sum \gamma_{i}(T) g_{i}(\rho)}, \quad \sigma(T)=\sum \frac{\gamma_{i}(T) \sigma_{i} g_{i}(\sigma)}{\sum \gamma_{i}(T) g_{i}(\sigma)},
$$

где сумма концентраций фаз $\gamma_{i}$ равна 1 , а конфигурационные параметры $g_{i}(\rho)$ и $g_{i}(\sigma)$ вдоль электрических (тепловых) токов имеют вид:

$$
g_{i}(\sigma)=\frac{3 \sigma}{A \sigma+(3-A) \cdot \sigma_{i}}, \quad g_{i}(\rho)=\frac{3 \rho}{A \rho+(3-A) \cdot \rho_{i}} .
$$
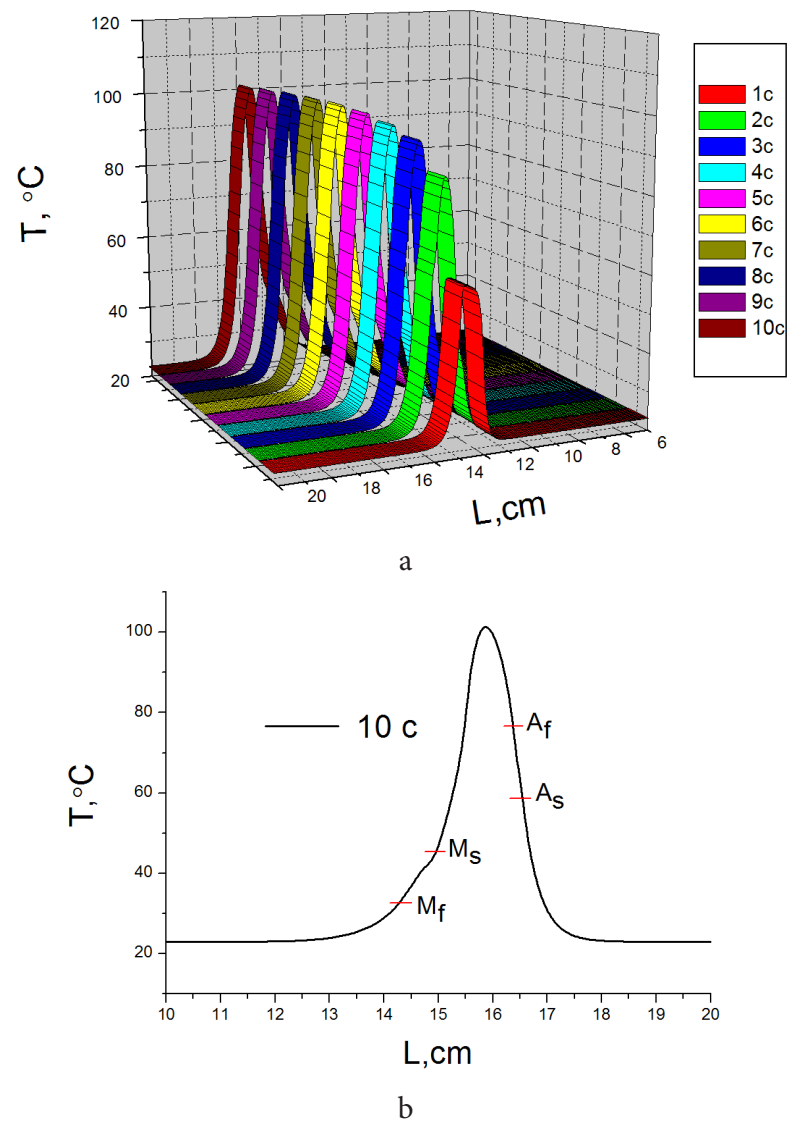

Puc. 1. (Color online) Распределение температуры вблизи зоны нагрева проволочного образца TiNi: за первые десять секунд (a); за десятую секунду (b).

Fig. 1. (Color online) Temperature distribution of the TiNi wire sample near the heating zone: (a) for the first ten seconds; (b) for the tenth second. 
Параметр А равен 0, 3 или 1 для случая параллельного и последовательного электрического соединения или для случая сферических включений, соответственно. Промежуточные значения $A(0<A<3)$ соответствуют интерполированной конфигурации включений по определенным направлениям, например, растянутые или сплюснутые эллипсоиды.

Из рассчитанной по формулам (1) - (6) зависимости величины термокинетической ЭДС от температуры в зоне нагрева следует, что при движении зоны нагрева с температурой ниже температуры начала обратного фазового перехода $\left(T<A_{н}\right)$, термокинетическая ЭДС в проволочном образце не возникает. Если температура в зоне нагрева равна или выше температуры конца обратного фазового перехода $\left(T>A_{\kappa}\right)$, то возникает термокинетическая ЭДС не превышающая 0,23 мВ.

При промежуточных значениях температуры в зоне нагрева $\left(A_{H} \leq T \leq A_{\kappa}\right)$ также будет возникать термокинетическая ЭДС, при этом, чем выше температура в указанном диапазоне тем, больше величина наводимой ЭДС (рис. 2).

Для подтверждения теоретических расчетов проведены экспериментальные исследования на проволочных образцах $\mathrm{Ti}-50$ ат.\% Ni длиной 400 мм (длина рабочей части 300 мм) и диаметром 0,6 мм. Предварительно образцы были отожжены при температуре $700^{\circ} \mathrm{C}$ в течение 30 минут на воздухе. Характеристические температуры мартенситных переходов определенные методом дифференциальной сканирующей калориметрии на DSC822 (METTLER TOLEDO), составили: $M_{\mu}=45^{\circ} \mathrm{C}, M_{\kappa}=33^{\circ} \mathrm{C}$, $A_{н}=59^{\circ} \mathrm{C}, A_{\kappa}=76^{\circ} \mathrm{C}$. Анализ зависимости теплового потока (регистрируемой на DSC822e) от температуры TiNi образцов указывает, что в образцах прямой фазовый переход осуществляется по схеме В2 $\rightarrow$ B19', обратный по схеме В19' $\rightarrow$ В2. Локальный участок нагрева перемещали вдоль образца поочередно в обоих направлениях с постоянной скоростью $0,44 \mathrm{~cm} / \mathrm{c}$, что обеспечивало на участке нагрева обратный фазовый переход $(\mathrm{M} \rightarrow \mathrm{A})$ и приводило к возникновению постоянной во времени термокинетической ЭДС (рис. 3). Знак наведенной термокинетической ЭДС зависит от направления движения зоны нагрева и изменяется на противоположный при изменении направления движения.

Экспериментальное значение термокинетической ЭДС, возникающей при движении зоны нагрева вдоль проволочного образца TiNi составляло до $0,2 \mathrm{MB}$, что хорошо согласуется с рассчитанным по формулам (1) - (6) значением 0,23 мB.

\section{3. Заключение}

Предложенные математические зависимости позволяют рассчитать величину термокинетической ЭДС в никелиде титана, при обратном фазовом превращении в зоне нагрева. Термокинетическая ЭДС возникает при достижении температуры в зоне нагрева температуры начала обратного фазового перехода $A_{н}$ и увеличивается до 0,23 мВ с повышением температуры до $A_{\kappa}$. Дальнейшее повышение температуры на величину термокинетической ЭДС не влияет.

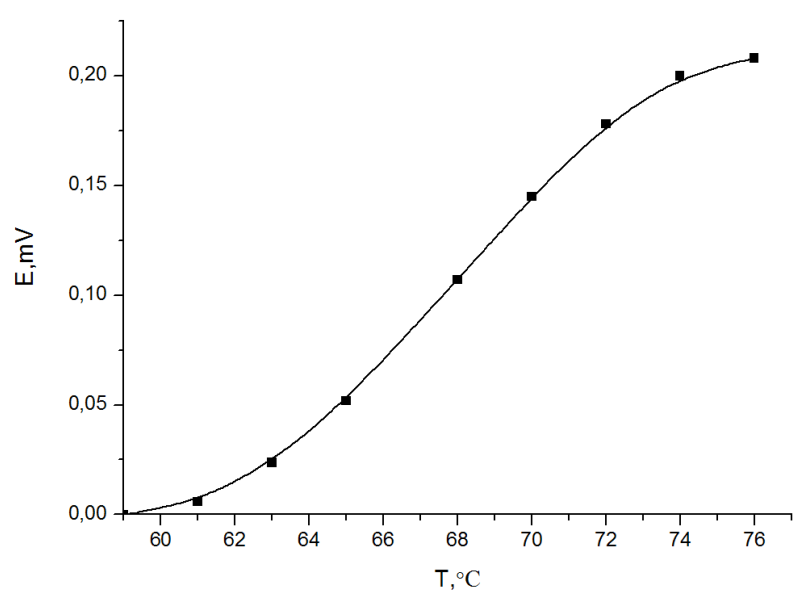

Рис. 2. Зависимость термокинетической ЭДС от температуры в зоне нагрева (расчет).

Fig. 2. Dependence of thermokinetic EMF on temperature of heating zone (calculation).

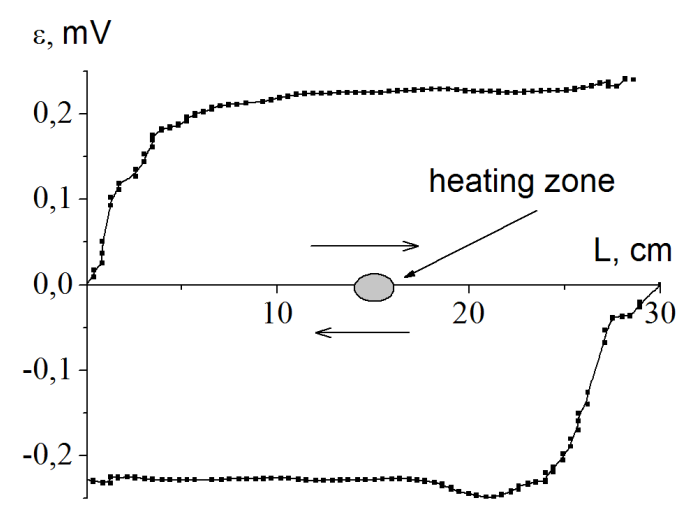

Рис. 3. Зависимость величины термокинетической ЭДС от местоположения зоны нагрева. Стрелками указано направление движения зоны нагрева

Fig. 3. Dependence of thermokinetic EMF on the location of heating zone. The arrows indicate the movement direction of the heating zone.

Экспериментально подтверждено, что в процессе перемещения участка нагрева $\left(T>A_{\kappa}\right)$ вдоль проволочного TiNi образца, находящегося в мартенситном состоянии, в нем возникает термокинетическая ЭДС до 0,2 мВ, что хорошо согласуются с результатами расчетов.

\section{Литература/References}

1. E. F. Furmakov. Fundamental problems of natural science. 1 (21), 377 - 378 (1999). (in Russian) [Е. Ф. Фурмаков. Фундаментальные проблемы естествознания. 1 (21), 377 - 378 (1999).]

2. V.V. Rubanik, V.V. Rubanik Jr., O.A. Petrova-Burkina. Letters on Materials. 2 (2), 71 (2012). (in Russian) [В.В. Рубаник, В.В. РубаникВ.В. мл., О.А. ПетроваБуркина. Письма о материалах. 2 (2), 71 (2012).]

3. V.V. Rubanik, V. V. Rubanik Jr., O.A. Petrova-Burkina. Materials of the 9th European Symposium on Martensitic Transformations «ESOMAT 2012».S.-Pb. (2012) p.40. 
4. V.V. Rubanik, V.V. Rubanik Jr., A.V. Lesota. Physical material: VII of the International School with elements of scientific school for young people. A collection of competitive reports. Tolyatti. (2016) p. 273-242. (in Russian) [В. В. Рубаник, В. В. Рубаник мл.,А. В. Лесота. Физическое материаловедение: VII Межд. школа с элементами научной школы для молодежи: сб. конкурсных докладов. Тольятти. (2016) с. 273 -242.]

5. C.E. Kulkova, D.V. Valuysky, I.Y. Smolin. Solid State Physics.43 (4), 706-713 (2001). (in Russian) [С.Е. Кулькова, Д.В. Валуйский, И.Ю. Смолин. Физика твердого тела. 43 (4), 706 - 713 (2001).]

6. L. I. Anatychuk, L.P. Bulat. Thermoelectric Phenomena under Large Temperature Gradients.Thermoelectrics Handbook: Macro to Nano-Structured Materials, CRC Press: New York, London, Tokyo, Chapter 3 (2005).

7. A.A. Golestaneh. Materials of the International Conference «Martensitic on Transformation». Massachusetts. (1979) p. 58.
8. V.E. Gyunter, V.N. Khodorenko. Nikelid titana. The medical material of new generation. Textbook. Tomsk, MITS. (2006) 55 p. (in Russian) [В.Э. Гюнтер, B.Н. Ходоренко. Никелид титана. Медицинский материал нового поколения. Томск, МИЦ. (2006) 55 с.

9. Vyunenko N. Materials of the International Conference «Advanced technologies and methods of control». Vitebsk. (2009) p. 384-399. (in Russian) [Вьюненко Ю.Н. Материалы конференции «Перспективные технологии и методы контроля». Витебск. (2009) с. $384-399$.

10. C. Liang, C.A. Rogers. Journal of Intelligent Material System and Structure, 1 (2) 207 - 234, (1990).

11. V. V. Shchennikov, S.V. Ovsyannikov, G.V. Vorontsov, V.V. phys. stat. sol. (b) 241, No.14 (2004).

12. S.V. Ovsyannikov, V.V. Shchennikov, I.A. Komarovskii, G.V. Vorontsov, I.V. Korobeynikov, V.V. Shchennikov Jr. Proceedings of the SPIE, Volume 7978, id. 79781W (2011). 